\title{
Robust surgical approach for cutaneous neurofibroma in neurofibromatosis type 1
}

\author{
Bahir H. Chamseddin, ${ }^{1}$ La'Nette Hernandez, ${ }^{1}$ Dezehree Solorzano, ${ }^{1}$ Juan Vega, ${ }^{1}$ and Lu Q. Le ${ }^{1,2,3}$
}

${ }^{1}$ Department of Dermatology, ${ }^{2}$ Comprehensive Neurofibromatosis Clinic, and ${ }^{3}$ Simmons Comprehensive Cancer Center, University of Texas (UT) Southwestern Medical Center, Dallas, Texas, USA.

BACKGROUND. Cutaneous neurofibromas (cNFs) are physically disfiguring, are painful, and cause extensive psychologic harm in patients with neurofibromatosis type 1 (NF1). There is currently no effective medical treatment, and surgical procedures are inaccessible to most NF1 patients globally.

\begin{abstract}
Although research is underway to find an effective medical treatment for cNF, there is an urgent need to develop a surgical approach that is accessible to all NF1 patients worldwide with the skill set and equipment found in most general medical office settings. Here, we present a robust surgical approach to remove cNFs that does not require a sterile surgical field, uses accessible clinical equipment, and can be performed by any health care provider, including family practitioners and physician assistants.
\end{abstract}

METHODS. In a prospective case series, patients with NF1 underwent this surgical procedure, which removes multiple cNFs. The Dermatology Life Quality Index was given to subjects before and after the procedure as a surrogate measurement of patient satisfaction.

RESULTS. Eighty-three tumors were removed throughout the body from 12 individuals. Examination at follow-up visits revealed well-healed scars without infection or adverse events, including aberrant scarring. Patient satisfaction with the procedure was high, with significant improvements in symptoms, daily activities, leisure, personal relationships, and treatment experience $(P=0.00062)$.

CONCLUSION. This study demonstrates a robust surgical approach to manage cNFs, which can be accessed worldwide by individuals with NF1 and performed by a wide variety of medical specialists with high clinical efficacy and patient satisfaction.

FUNDING. The Burroughs Wellcome Fund, the National Cancer Institute of the NIH, the Neurofibromatosis Therapeutic Acceleration Program, the NF1 Research Consortium Fund, and the Giorgio Foundation.

Conflict of interest: The authors have declared that no conflict of interest exists.

Copyright: (c) 2019 American Society for Clinical Investigation

Submitted: March 15, 2019

Accepted: April 23, 2019

Published: June 6, 2019.

Reference information: /CI Insight. 2019;4(11):e128881. https://doi. org/10.1172/jci.insight.128881

\section{Introduction}

Neurofibromatosis type 1 (NF1) is one of the most common autosomal dominant genetic disorders, affecting 1 in 3000 live births, and is found worldwide independent of sex, race, or geographic location (1). It results from loss of the NF1 tumor suppressor gene leading to extensive tumor formation throughout the body, and a hallmark of the disorder is multiple dermal or cutaneous neurofibromas (cNFs) (2). About 30\% of individuals with NF1 also develop plexiform neurofibroma, a Schwann cell tumor along the internal nerve plexuses that has around 10\% lifetime risk for malignant transformation (1). On the other hand, the cNFs are present in virtually all patients with NF1. They are exclusively located in the cutaneous dermis layer and are not prone to malignancy. Despite their benign physiology, patients with NF1 attribute the cNFs as their greatest medical burden because of their physical disfigurement (3). Patients with NF1 can develop thousands of cNFs that are $2 \mathrm{~mm}$ to $3 \mathrm{~cm}$, soft, skin-colored nodules covering the face, trunk, and extremities. CNFs are the primary source of chronic physical symptoms, such as pain and itching, and emotional distress given a greater burden of cNFs strongly correlates to negative quality of life and self-image (4).

The cNF comprises diverse cellular components, including Schwann cells, fibroblasts, macrophages, mast cells, and blood vessels, in a collagenous matrix within the dermis (5-7). Although their pathophysiology is 

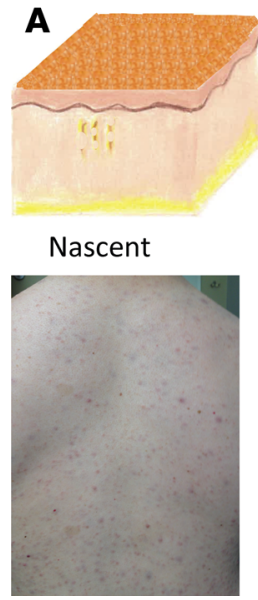

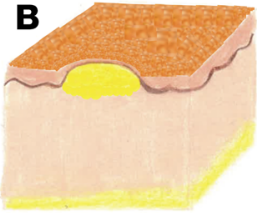

Flat

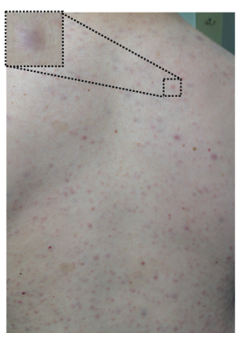

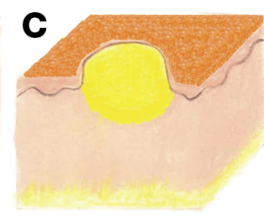

Sessile

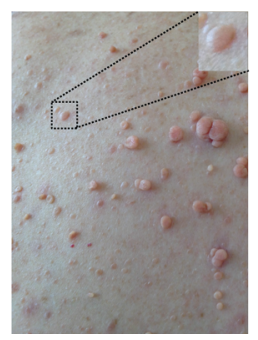

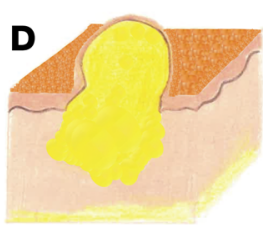

Globular

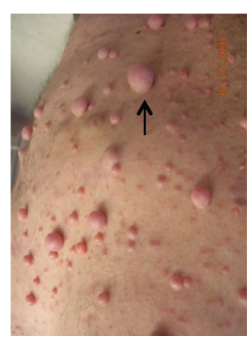

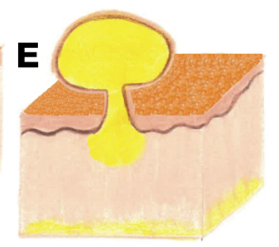

Peduncular

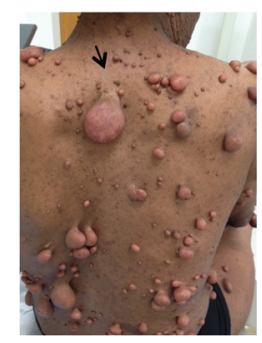

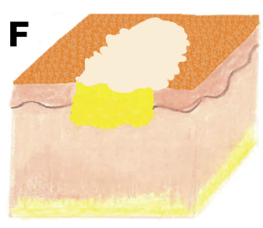

Post-Operative Scar

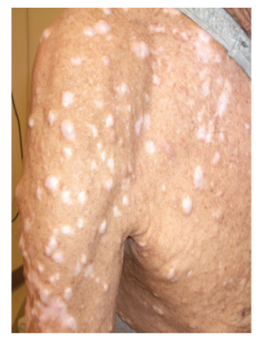

Figure 1. Clinical stages/evolution of cNFs and treatment. (A) Nascent, a dormant stage undetectable without instrumentation. (B) Flat, thinning or hyperpigmentation at the surface of the skin. (C) Sessile, tumor raised with an apex. (D) Globular, 20- to 30-mm base with comparable height and globular shape. (E) Peduncular, a stalk connects the portions above and below the skin. (F) Scars from $\mathrm{CO}_{2}$ laser treatment in cNFs' final stage (permanent).

unknown, cNFs are affected by genetic variation of NF1, the skin microenvironment, and hormones and may grow in evolving stages (Figure $1 ;$ refs. 8,9 ). They are classified by stage according to appearance: the nascent stage detected only through ultrasound or other forms of imaging, the flat stage depicted by thinning or hyperpigmentation at the surface of the skin, the sessile stage with a raised papule, the globular stage with a 20- to 30-mm base with comparable height, and the pedunculated stage, which extrudes its dermal contents through a visible stalk connecting the portions above and below the skin $(9,10)$. In all stages, considerable mass is located within the deeper dermis layer; thus, typical shaving or electrodessication treatments, which target only the visible projection, may lead to tumor regrowth and collagen scar formation (Figure 1; refs. 11, 12). Currently, there is no available medical treatment for cNFs. Physical removal remains the mainstay of treatment, primarily focused on surgical excision with primary closure by dermatologists or general and plastic surgeons $(13,14)$. Unfortunately, this method may be inaccessible to a majority of the global population because of the requirements of surgical expertise, a sterile field, and general anesthesia. $\mathrm{CO}_{2}$ lasers have been developed since the 1980s to remove hundreds of tumors at one time but pose high risk of hypopigmented or hypertrophic scarring (Figure 1) and continue to remain largely inaccessible to the population with NF1 because of lack of specialized training and equipment $(15,16)$.

Because of the dearth of accessibility to treatment for patients with NF1 worldwide, we offer a robust surgical approach for management of cNFs that can be performed in an outpatient setting with equipment available to most general medical clinics and can be performed by almost all medical providers, including family practitioners, physician assistants, and nurse practitioners. In this study, we recognize all adverse events associated with the procedure and quantify patient satisfaction.

\section{Results}

Twelve patients with NF1 gave informed consented and underwent the surgical procedure (Figure 2). The average age of the subjects was 46.6 (SD; ref. 17), 10 (80\%) of the subjects were female, and 11 (92\%) were white, with 1 African American. In the entire study, $83 \mathrm{cNFs}$ were removed and were an average size of $1 \mathrm{~cm}$ (SD, 0.35). An average of 6.9 (range: 1-10) cNFs were removed each operation. Thirty-one (37.3\%) cNFs removed were located on the upper extremity, $29(35 \%)$ on the trunk, $11(13.3 \%)$ on the head and neck, $10(12 \%)$ on the abdomen, and 2 (2.4\%) on the lower extremity. This surgical technique is based on the biology and anatomy of the $\mathrm{cNF}$, where a large portion of the tumor is in the dermis. Therefore, a critical component to this procedure is further removal of the mass within the deeper dermis. This is accomplished after shaving off the outer projection of the neurofibroma by grasping the remaining mass with forceps, lifting the mass outward, and using the dermablade (or razor blade) to remove the pale, collagenous tumor. The open lesion is then closed by 1 or 2 interrupted stitches depending on its size. The whole procedure can be done in less than 2 minutes per tumor. There were no complications during the operations, including excessive pain or problems with local anesthesia. 


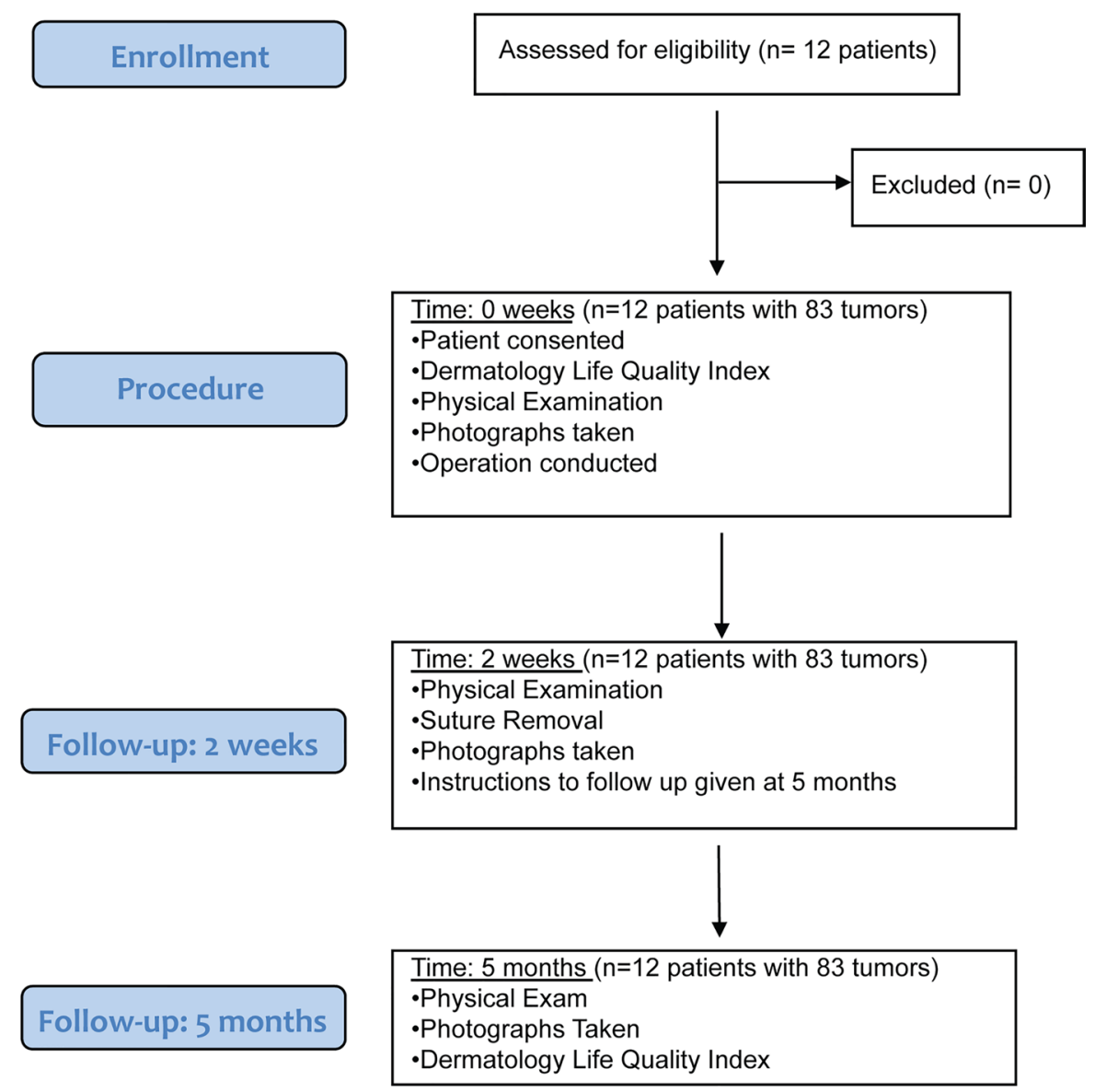

Figure 2. Flow diagram for operation.

Initial follow-up was 14 days and extended follow-up averaged 5 months. Representative photographs of the tumors before and after the procedure are depicted in Figure 3. Examination at the extended follow-up revealed 1 site with hypertrophic scar formation (1.2\%) (Supplemental Figure 1; supplemental material available online with this article; https://doi.org/10.1172/jci.insight.128881DS1) and 10 sites with postinflammatory hyperpigmentation (12\%) (Supplemental Figure 2), but no other complications were noted, including skin infection, tumor regrowth, hypopigmentation, or keloid formation (Table 1).

The Dermatology Life Quality Index (DLQI) (17) was administered to all 12 patients before the surgical procedure and after the extended follow-up visit. Every question was answered to completion, and the 10-question survey took a maximum of 5 minutes to complete for each person. The initial DLQI average and total scores were 9.83 and 118 , respectively, which improved significantly at the extended follow-up visit to 1.83 and 22 , respectively $(P=0.00062)$. The individual DLQI dimensions before and after the surgical procedure are displayed in Table 2 and Supplemental Figure 3. The index of symptoms and feelings dropped from $3.1(\mathrm{SD}, 1.1)$ to $1.1(\mathrm{SD}, 0.9)(P<0.0001)$, daily activities from $2.8(\mathrm{SD}, 1.2)$ to $0.5(\mathrm{SD}, 0.4)$ $(P<0.0001)$, and leisure activities from $1.7(\mathrm{SD}, 1.1)$ to $0.3(\mathrm{SD}, 0.3)(P<0.0001)$. Personal relationships and treatment efficacy dropped from average scores of $1.1(\mathrm{SD}, 0.8)$ and $0.6(\mathrm{SD}, 0.8)$ to 0 , respectively. Although work and school functioning improved from $0.6(\mathrm{SD}, 1.2)$ to 0 , the results were not significant.

\section{Discussion}

The goal of the present report is to propose a potentially novel technique for removing cNFs that is accessible to the global population with NF1. The procedure described herein is considered accessible by using inexpensive medical equipment present in most outpatient general clinical settings, employing a nonsterile technique, and following a low-risk procedure, which can be performed by most health care providers. The procedure yields favorable cosmetic results and improves quality of life in patients with NF1. 

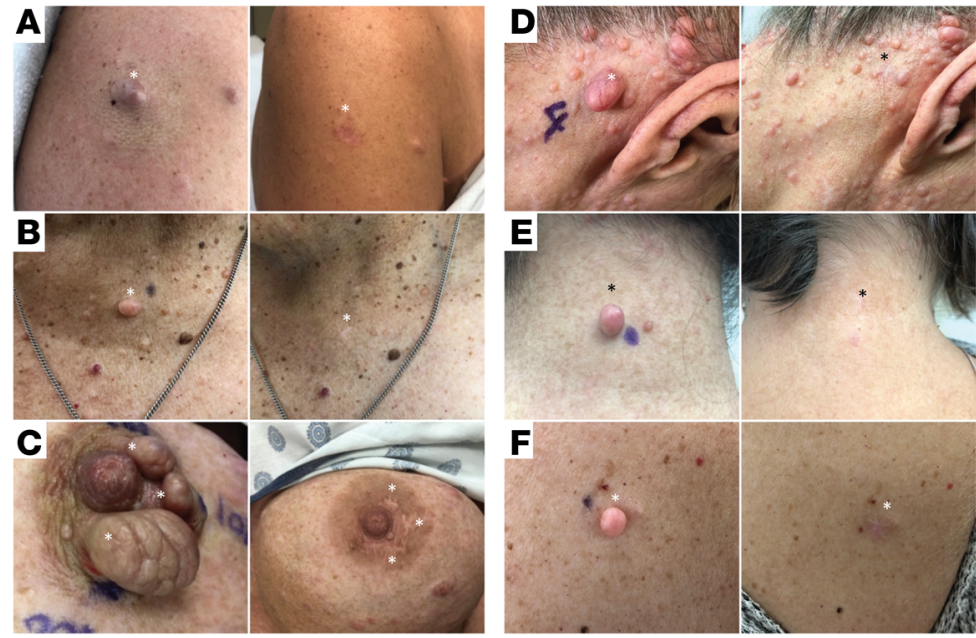

Figure 3. Cosmetic outcomes of cNF removal using described procedure at 5 months. Representative photos (left) before and (right) after tumor removal at the (A) right shoulder, (B) chest, (C) left nipple, (D) posterior auricular, (E) posterior neck, and $(\mathbf{F})$ back.

Definitive treatment for $\mathrm{NF}$ is a major obstacle for NF1 patients. Patients with NF1 are often affected with other medical problems, including bony deformities, malignant neoplasms, and learning disability, which could affect their access to treatment (18). Additionally, the type and size of cNFs, location of the tumor, and patient demographics must be considered for different treatment modalities. Excisional operations performed by general and plastic surgeons have the capability of removing extremely large cNFs or hundreds of cNFs in one operation with exceptional reconstructive results $(13,14,19)$. Unfortunately, this method mandates trained surgical specialists, requires a sterile surgical field, and runs a high-risk side-effect profile with use of general anesthesia, higher costs, and potential requirement of postoperative hospitalization $(13,14)$. Dermatologists typically will conduct excisional removal using local anesthesia to manage cNFs in an outpatient setting. It has a lower risk profile and cost to the patients with NF1 than operative surgery, yet the necessity for a longer excision to remove the cNFs limits postoperational cosmetic outcome (20). In addition, this traditional excision can remove only a few tumors per session because of the time constraints. The procedure herein targets $\mathrm{cNF}$ anatomy by selectively removing the dermal component, which prevents tumor regrowth and ultimately yields more favorable postprocedural cosmetic outcomes for the patients by shortening the excision length. Local anesthesia with $1 \%$ lidocaine with epinephrine 1:100,000 is the agent of choice for skin surgery and is generally safe to use with maximum dose of $40 \mathrm{cc}(\mathrm{ml})$ per session or $7 \mathrm{mg} / \mathrm{kg}$ total $(21,22)$. Cautionary use is advised when using epinephrine to the fingers, toes, nose, or penis to avoid a theoretical complication of vasoconstriction and digital gangrene despite evidence largely in support of its safety in the digits $(23,24)$. Rare systemic toxicity from lidocaine may include hypotension, bradycardia, or systemic allergic reaction (22). For wound closure, the use of surgical glue or staples could replace sutures to expedite the removal process of cNFs and to minimize the resultant skin tension from sutures after removal of clustered cNFs. Staples could be considered for larger neurofibromas in areas of less cosmetic concern (i.e., unexposed skin), with benefits of quicker operational time and lesser infection risks, but are prone to cause greater postoperative pain than traditional sutures (25). Surgical glue should be implemented only on small lesions under no tension postoperatively. This modality may offer less scarring but additional risk of allergic reaction to adhesives and should not be used in high-moisture areas, such as the axillae and perineum, or highly mobile areas, including hands, feet, and joints (26).

The $\mathrm{CO}_{2}$ laser is a commonly used tool for treating $\mathrm{cNF}$ by rapidly heating and vaporizing the intracellular water, which leads to destruction of tissue (15). The advantage of this technique includes the ability to treat hundreds of tumors in proximity to one another and can be performed in the operating room or outpatient clinical setting with high patient satisfaction $(15,27,28)$. The $\mathrm{CO}_{2}$ laser technology is, however, unavailable to most global clinical settings, and specialized training is required to operate the machinery. Local infection rate has been reported up to $15 \%$, and scarring is a frequently observed 
Table 1. Demographics and operation outcomes of patients who underwent cNF removal

\begin{tabular}{|c|c|c|c|c|c|c|c|}
\hline Age & Patient & Sex & Ethnicity & Location & No. of tumors & Tumor type & Complications \\
\hline \multirow{2}{*}{$20-29$} & 1 & M & AA & Trunk & 1 & Globular & Hypertrophic scar \\
\hline & & & & Upper extremity & 2 & Globular & None \\
\hline \multirow{4}{*}{$30-39$} & 2 & $\mathrm{~F}$ & W & Trunk & 6 & Globular (5), pedunculated & None \\
\hline & & & & Upper extremity & 1 & Globular & None \\
\hline & 3 & $\mathrm{~F}$ & W & Abdomen & 1 & Sessile & None \\
\hline & & & & Upper extremity & 5 & Globular (2), sessile (3) & None \\
\hline \multirow{7}{*}{$40-49$} & & & & Trunk & 1 & Globular & None \\
\hline & & & & Upper extremity & 3 & Sessile & None \\
\hline & 6 & $\mathrm{~F}$ & W & Head and neck & 1 & Pedunculated & None \\
\hline & & & & Trunk & 6 & Globular (5), sessile & None \\
\hline & & & & Upper extremity & 3 & Pedunculated, sessile (2) & None \\
\hline & & & & Trunk & 3 & Globular & None \\
\hline & & & & Upper extremity & 2 & Globular & None \\
\hline \multirow{2}{*}{$50-59$} & 9 & $\mathrm{~F}$ & W & Trunk & 4 & Globular (2), pedunculated (2) & None \\
\hline & & & & Upper extremity & 4 & Globular & None \\
\hline \multirow{4}{*}{$60-69$} & 10 & $\mathrm{~F}$ & W & Head and neck & 4 & Globular & None \\
\hline & & & & Trunk & 4 & Globular (1), pedunculated (3) & None \\
\hline & & & & Upper extremity & 2 & Globular & None \\
\hline & 11 & $\mathrm{~F}$ & W & Upper extremity & 8 & Globular (4), sessile (4) & None \\
\hline \multirow{3}{*}{$70-79$} & 12 & $\mathrm{~F}$ & W & Head and neck & 3 & Globular (2), pedunculated & None \\
\hline & & & & Trunk & 2 & Globular, pedunculated & None \\
\hline & & & & Upper extremity & 1 & Globular & None \\
\hline
\end{tabular}

M, male; F, female; AA, African American; W, white.

side effect $(15,27-29)$. Minimally invasive photocoagulation, including Er:YAG or Nd:YAG lasers, has been successfully implicated as a recent treatment option for small- to medium-sized cNFs, with complications such as postinflammatory hyperpigmentation occurring in $4 \%$ of tumors, yet the access is limited to large academic centers $(30,31)$. Other destructive methods, including electrodessication and radiofrequency ablation, have shown high patient satisfaction outcomes 6 months postprocedure but should be considered as second-line therapies because of the high risks of aberrant scarring and hypopigmentation associated with the techniques $(10,31,32)$.

This study provides a robust, accessible method to remove multiple cNFs per visit that can have a strongly positive impact on quality of life for patients with NF1. The DLQI survey was specifically chosen for its ability to assess $\mathrm{CNF}$ removal impact directly on physical and psychosocial factors related to the skin, whereas the Impact of NF1 on Quality of Life survey, an important tool to assess quality of life for NF1 patients, assesses nondermatologic features of the disease, including vision problems, gait abnormalities, and mental status changes that will not reflect the outcomes of this procedure $(4,33)$. Symptoms of itchiness, soreness, or pain and feelings of self-consciousness were among the highest reasons for poor quality of life preoperatively; however, the described procedure significantly diminished the magnitude of these dimensions and most others based on the DLQI. Removal of cNFs with this technique could be implemented as a low-risk therapy for both symptomatic and aesthetically troublesome neurofibromas in patients with a limited number of cNFs. It is important to assess the potential risks of complications, including hypertrophic scarring, keloid formation, and postinflammatory pigmentation. Keloids, abnormal collagen scarring formations, may occur with higher frequency in African American patients or patients with a history of keloid formation (34). Thus clinicians should warn such individuals about these risks when performing any surgical procedure in this context. 
Table 2. DLQI averages among patients who had cNFs removed

\begin{tabular}{lccc}
\hline Dimension & Preop mean (SD) & Postop mean (SD) & $\boldsymbol{P}$ value \\
Symptoms and feelings (maximum: 6) & $3.1(1.1)$ & $1.1(0.9)$ & $<\mathbf{0 . 0 0 1}$ \\
Daily activities (maximum: 6) & $2.8(1.2)$ & $0.5(0.4)$ & $<\mathbf{0 . 0 0 0 1}$ \\
Leisure (maximum: 6) & $1.7(1.1)$ & $0.3(0.3)$ & $<\mathbf{0 . 0 0 0 1}$ \\
Personal relationships (maximum: 6) & $1.1(0.8)$ & $0(0)$ & $<\mathbf{0 . 0 0 0 1}$ \\
Work and school (maximum: 3) & $0.6(1.2)$ & $0(0)$ & 0.0967 \\
Treatment (maximum: 3) & $0.6(0.8)$ & $0(0)$ & $\mathbf{0 . 0 1 8 3}$ \\
$n=12$ patients. Statistical test: 2-tailed, paired $t$ test. & & \\
\hline
\end{tabular}

Postinflammatory hyperpigmentation (PIH) more commonly occurs in people with Fitzpatrick skin types IV-VI and people of Asian descent because of activation of melanocytes after trauma and dermal procedures (35). Patients with PIH should be reassured of the benign nature of the condition and that spontaneous resolution occurs after months, or potentially years (35). If PIH is an issue, it can be managed postoperatively with UV protection, topical steroids, or retinoids and lightening cream hydroquinone (36). Future studies should stratify treatment strategies based on these risks, skin types, and location of cNFs (31).

Physicians of different subspecialty training background and advanced practitioners can use this simple technique to remove cNFs for their patients with NF1, but caution should be used to ensure safety. cNFs must be accurately identified from other potential differentiating soft tissue masses, including plexiform neurofibromas, dermatofibroma protuberans, or dermatofibromas. Because of the superficial nature of this operation and low risk for complications, the procedure is deemed "low risk" and thus may be performed by practitioners licensed in the United States with credentialing for performing these operations. We provide Supplemental Video 1 showing step by step the whole procedure for additional training. Patients on anticoagulation therapy or at risk of bleeding should continue to undergo the procedure given the low risk of the operation. Additionally, the cost of the operation would be comparable to a tangential biopsy (billing code 11102 for the first lesion and 11103 for additional lesions). There is a need for more accurate billing codes for $\mathrm{CNF}$ resection as a priority for future work given the physical disfigurement and psychologic harm of the lesions.

There are a several of limitations to this study to address. The procedure was performed at a single institution with a relatively small sample size, thus affecting generalizability. Selection bias may have
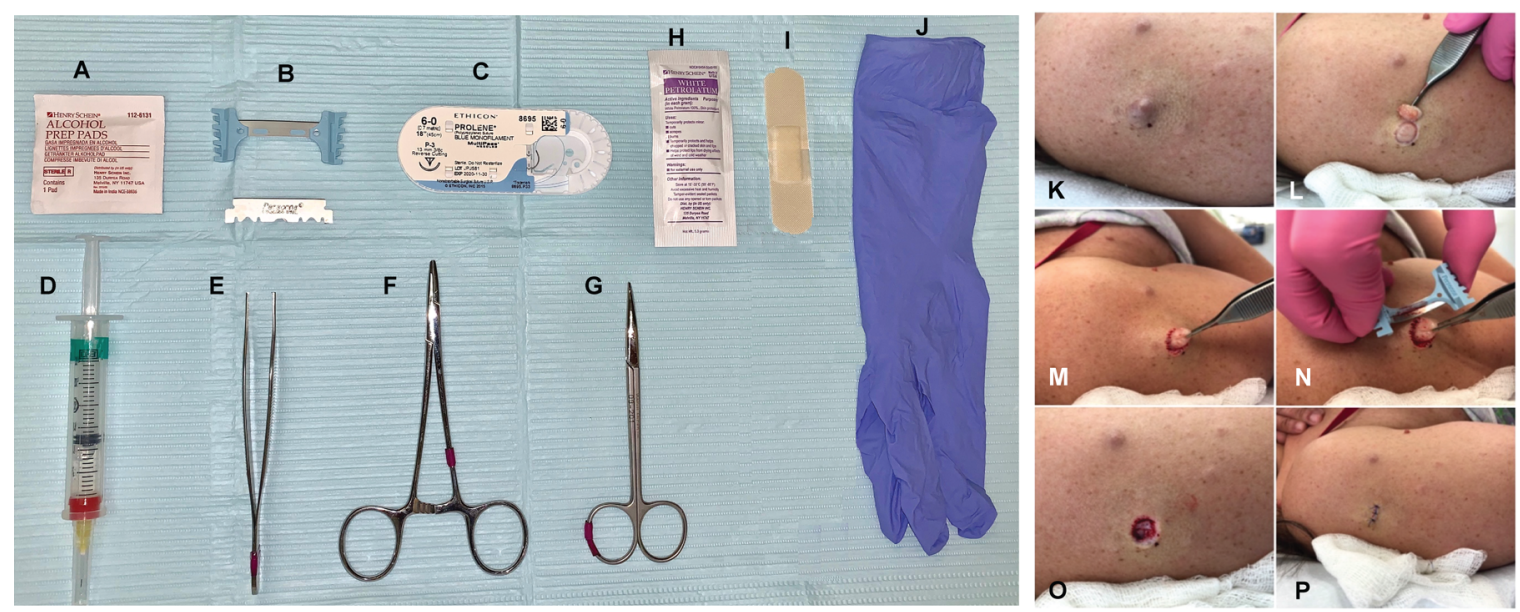

Figure 4.

Materials and visual procedure sequences. (A) Isopropyl alcohol (70\%) pads. (B) Dermablade or razor blade. (C) Suture. (D) Local anesthetic (1\% lidocaine hydrochloride and epinephrine 1:100,000). (E) Forceps. (F) Needle driver. (C) Curved/straight Mayo scissors. (H) Petrolatum. (I) Bandage. (J) Nonsterile gloves. (K) cNF, sessile. (L) Tumor removed at skin level with dermablade or razor blade. (M) Forceps grasp and exposure of dermal component. (N) Removal of dermal component with dermablade or razor blade. (0) Site with completely removed cNF. (P) Skin sutured. 
influenced the quality-of-life improvements given the individuals who were most unhappy with their skin elected to undergo the surgery. The majority of subjects were white, and postprocedural outcomes, including PIH, may not accurately reflect outcomes for the entire population affected by NF1.

\section{Methods}

Patients over the age of 18 with a diagnosis of NF1 established by the NIH neurofibromatosis guidelines were recruited at the Comprehensive Neurofibromatosis Clinic at UT Southwestern Medical Center, Dallas, Texas, between February 1, 2018, and June 30, 2018, in a prospective clinical case series (18). The procedure, risks and benefits, and importance of postoperative care were described to the patients before they provided consent. Preoperative assessment included a skin examination and the collection of sociodemographic characteristics.

Materials. The surgical procedure requires isopropyl alcohol (70\%) pads, local anesthetic (1\% lidocaine hydrochloride and epinephrine 1:100,000), dermablade or razor blade, forceps, sutures, needle driver, curved/straight Mayo scissors, nonsterile gloves, adhesive bandage, and petrolatum ointment. Required materials are depicted in Figure 4. Surgical glue or stapler may replace suturing supplies for an expedient technique on smaller cNFs.

Procedure. First, we disinfected the $\mathrm{cNF}$ with alcohol wipes and applied local anesthesia with $1 \%$ lidocaine/epinephrine 1:100,000 to the neurofibroma and surrounding skin. After several minutes to allow anesthesia to take effect, a dermablade (or razor blade) was used to shave off the outer projection of the neurofibroma level to the surrounding skin. Of note, the tumor within the deeper dermis may naturally project out because of surrounding tension from the skin. A critical component to this procedure is further excision or removal of the mass within the deeper dermis. This was accomplished by grasping the remaining mass with forceps, lifting the mass outward, and using the dermablade (or razor blade) to remove the pale, collagenous tumor. The open lesion was then closed by 1 or 2 interrupted stitches depending on its size. The closed wounds were covered with white petroleum and an adhesive bandage. The subjects were educated to continue application of petroleum ointment every day until the 2-week follow-up for suture removal. No antibiotics, oral or topical, were given before or after the procedure, and clean, nonsterile gloves were used throughout the procedure. The procedure can be viewed in Supplemental Video 1, and it takes less than 2 minutes to remove each tumor.

Demographic features of the subjects, such as age, sex, and ethnicity, and the tumor properties, including size and location, were recorded on the day of the procedure. The patients had up to $10 \mathrm{cNFs}$ removed per session with follow-up in the clinic for suture removal in 2 weeks, which included wound check. The subjects returned for an extended follow-up visit at least 4 months from the procedure date to analyze the following outcomes: infections, scar assessment, keloid formation, hypo/hyperpigmentation, and other adverse events.

The DLQI (17), a survey that assesses patients' satisfaction with their skin, was administered to each patient before the procedure and at the extended follow-up. The DLQI is a questionnaire containing 10 questions, each with a response of "not at all," "a little," "a lot," or "very much" with corresponding scores of $0,1,2$, and 3, respectively (33). Higher scores are indicative of worsening quality of life. Questions 1 and 2 are representative of the patient's symptoms and feelings, questions 3 and 4 examine daily activities, questions 5 and 6 indicate impact on leisure activities, question 7 analyzes problems with work and school, questions 8 and 9 indicate personal relationship effect, and question 10 examines treatment efficacy.

Statistics. Statistical analysis included continuous data presented as means with SDs and categorical data as counts with percentages. A 2-tailed, paired $t$ test was used to compare the outcomes of the DLQI. $P \leq 0.05$ was considered significant.

Study approval. The UT Southwestern Institutional Review Board approved this study, and all patients provided signed IRB consent documents and Health Insurance Portability and Accountability Act privacy forms before inclusion in the study. Written informed consent was provided for pictures appearing in the manuscript.

\section{Author contributions}

BHC acquired data, analyzed data, and drafted the manuscript. LH, DS, and JV acquired data. LQL designed the research study, acquired data, analyzed data, and drafted and reviewed the manuscript. 


\section{Acknowledgments}

LQL holds a Career Award for Medical Scientists from the Burroughs Wellcome Fund and the Thomas L. Shield, M.D. Professorship in Dermatology and is supported by funding from the National Cancer Institute of the NIH (grant number R01 CA166593), the Neurofibromatosis Therapeutic Acceleration Program, the NF1 Research Consortium Fund, and the Giorgio Foundation.

Address correspondence to: Lu Q. Le, Department of Dermatology, 5323 Harry Hines Blvd., Dallas, Texas 75390-9069, USA. Phone: 214.648.5781; Email: Lu.Le@UTSouthwestern.edu.

1. Williams VC, Lucas J, Babcock MA, Gutmann DH, Korf B, Maria BL. Neurofibromatosis type 1 revisited. Pediatrics. 2009;123(1):124-133.

2. Le LQ, Shipman T, Burns DK, Parada LF. Cell of origin and microenvironment contribution for NF1-associated dermal neurofibromas. Cell Stem Cell. 2009;4(5):453-463.

3. Granström S, Langenbruch A, Augustin M, Mautner VF. Psychological burden in adult neurofibromatosis type 1 patients: impact of disease visibility on body image. Dermatology (Basel). 2012;224(2):160-167.

4. Ferner RE, et al. Evaluation of quality of life in adults with neurofibromatosis 1 (NF1) using the Impact of NF1 on Quality Of Life (INF1-QOL) questionnaire. Health Qual Life Outcomes. 2017;15(1):34.

5. Jouhilahti EM, et al. The development of cutaneous neurofibromas. Am J Pathol. 2011;178(2):500-505.

6. Ortiz-Hidalgo C, Weller RO. Peripheral nervous system. In: Sternberg SS, ed. Histology for Pathologists. 2nd ed. Philadelphia, Pennsylvania, USA: Wolters Kluwer; 1997:11.

7. Le LQ, Kesterson RA, Guttmann DH. Defining the research landscape for dermal neurofbromas. Oncology Times. 2016;38(18):14-15.

8. Allaway RJ, et al. Cutaneous neurofibromas in the genomics era: current understanding and open questions. Br J Cancer. 2018;118(12):1539-1548.

9. Riccardi VM. Translational genetics, genomics: the fundamental nature of NF1 neurofibromas. J Translational Genet Genomics. 2017:1-12.

10. Ortonne N, et al. Cutaneous neurofibromas: Current clinical and pathologic issues. Neurology. 2018;91(2 suppl 1):S5-S13.

11. Kim SH, Roh SG, Lee NH, Yang KM. Radiofrequency ablation and excision of multiple cutaneous lesions in neurofibromatosis type 1. Arch Plast Surg. 2013;40(1):57-61.

12. Roberts AH, Crockett DJ. An operation for the treatment of cutaneous neurofibromatosis. Br J Plast Surg. 1985;38(2):292-293.

13. Bromley GS, Sherman JE, Goulian D Jr. Neurofibromatosis-distribution of lesions and surgical treatment. Ann Plast Surg. 1982;8(4):272-276.

14. Yuan SM, et al. Surgical management of giant neurofibroma in soft tissue: a single-center retrospective analysis. Int J Clin Exp Med. 2015;8(4):5245-5253.

15. Becker DW. Use of the carbon dioxide laser in treating multiple cutaneous neurofibromas. Ann Plast Surg. 1991;26(6):582-586.

16. Roenigk RK, Ratz JL. CO2 laser treatment of cutaneous neurofibromas. J Dermatol Surg Oncol. 1987;13(2):187-190.

17. Finlay AY, Khan GK. Dermatology Life Quality Index (DLQI) - a simple practical measure for routine clinical use. Clin Exp Dermatol. 1994;19(3):210-216.

18. Ferner RE, et al. Guidelines for the diagnosis and management of individuals with neurofibromatosis 1 . JMed Genet. 2007;44(2):81-88.

19. Pailheret JP. [Plastic surgery in benign cutaneous manifestations of von Recklinghausen's disease]. Chirurgie. 1990;116(45):368-372.

20. Onesti MG, Carella S, Spinelli G, Scuderi N. The megasession technique for excision of multiple neurofibromas. Dermatol Surg. 2010;36(9):1488-1490.

21. Lawrence C. Drug management in skin surgery. Drugs. 1996;52(6):805-817.

22. Becker DE, Reed KL. Local anesthetics: review of pharmacological considerations. Anesth Prog. 2012;59(2):90-102.

23. Denkler K. A comprehensive review of epinephrine in the finger: to do or not to do. Plast Reconstr Surg. 2001;108(1):114-124.

24. Krunic AL, Wang LC, Soltani K, Weitzul S, Taylor RS. Digital anesthesia with epinephrine: an old myth revisited. J Am Acad Dermatol. 2004;51(5):755-759.

25. Iavazzo C, Gkegkes ID, Vouloumanou EK, Mamais I, Peppas G, Falagas ME. Sutures versus staples for the management of surgical wounds: a meta-analysis of randomized controlled trials. Am Surg. 2011;77(9):1206-1221.

26. Bruns TB, Worthington JM. Using tissue adhesive for wound repair: a practical guide to dermabond. Am Fam Physician. 2000;61(5):1383-1388.

27. Ostertag JU, Theunissen CC, Neumann HA. Hypertrophic scars after therapy with $\mathrm{CO}_{2}$ laser for treatment of multiple cutaneous neurofibromas. Dermatol Surg. 2002;28(3):296-298.

28. Méni C, et al. Treatment of neurofibromas with a carbon dioxide laser: a retrospective cross-sectional study of 106 patients. Dermatology (Basel). 2015;230(3):263-268.

29. Chiang YZ, Al-Niaimi F, Ferguson J, August PJ, Madan V. Carbon dioxide laser treatment of cutaneous neurofibromas. Dermatol Ther (Heidelb). 2012;2(1):7.

30. Kriechbaumer LK, Susani M, Kircher SG, Distelmaier K, Happak W. Comparative study of $\mathrm{CO}_{2}$ - and Er:YAG laser ablation of multiple cutaneous neurofibromas in von Recklinghausen's disease. Lasers Med Sci. 2014;29(3):1083-1091.

31. Verma SK, et al. Considerations for development of therapies for cutaneous neurofibroma. Neurology. 2018;91(2 suppl 1):S21-S30.

32. Levine SM, Levine E, Taub PJ, Weinberg H. Electrosurgical excision technique for the treatment of multiple cutaneous lesions in neurofibromatosis type I. J Plast Reconstr Aesthet Surg. 2008;61(8):958-962. 
33. Lewis V, Finlay AY. 10 years experience of the Dermatology Life Quality Index (DLQI). J Investig Dermatol Symp Proc. 2004;9(2):169-180.

34. Mofikoya BO, Adeyemo WL, Abdus-salam AA. Keloid and hypertrophic scars: a review of recent developments in pathogenesis and management. Nig Q J Hosp Med. 2007;17(4):134-139.

35. Davis EC, Callender VD. Postinflammatory hyperpigmentation: a review of the epidemiology, clinical features, and treatment options in skin of color. J Clin Aesthet Dermatol. 2010;3(7):20-31.

36. Sofen B, Prado G, Emer J. Melasma and post inflammatory hyperpigmentation: management update and expert opinion. Skin Therapy Lett. 2016;21(1):1-7. 